\title{
Quasilocal mass and multipole expansion in scalar-tensor gravity
}

\author{
Valerio Faraoni, * and Jeremy Côté ${ }^{1, \dagger}$ \\ ${ }^{1}$ Department of Physics 8 Astronomy, Bishop's University, \\ 2600 College Street, Sherbrooke, Québec, Canada J1M 1Z7
}

\begin{abstract}
A generalization of the Hawking-Hayward quasilocal mass to scalar-tensor gravity is compared, in vacuo and for asymptotically flat stationary geometries, with a recent multipole expansion of the gravitational field. The quasilocal mass seen at spatial infinity coincides with the monopole term, lending credibility to this construct.
\end{abstract}

\section{INTRODUCTION}

There is little doubt that general relativity (GR) is not the final theory of gravity, since it must break down at spacetime singularities, which must be somehow cured, and GR cannot be renormalized without altering it. All attempts to quantize gravity generate modifications to it in the form of higher order derivatives in the field equations or curvature terms, non-local terms, or extra fields in the action 1] 1] What is more, perhaps we are already observing modifications of GR in the present cosmic acceleration that the standard $\Lambda$ CDM model of cosmology tries to fit into GR by invoking a completely ad hoc dark energy 3]. Maybe the acceleration is due to deviations from GR at large scales [4], an idea that has raised much interest and has led to the so-called $f(\mathcal{R})$ gravity (see [3, 5] for reviews). This class of theories is a subfamily of the broader scalar-tensor gravity that we consider here [6, 7], and which generalizes the original Brans-Dicke proposal 8]. There is currently a sustained theoretical and experimental effort to test gravity at multiple scales, in various regimes, and using various physical phenomena $[9]$ [16].

The notion of mass-energy in the relativistic physics of gravitating systems is complicated, because it must include gravitational energy. However, due to the equivalence principle 9], it is impossible to localize the energy of the gravitational field, which can be eliminated locally by changing to the frame of a freely falling observer. As the next best option, researchers have resorted to the use of quasilocal notions of energy, i.e., integral quantities computed over compact regions of space. The Hawking-Hayward mass construct [17] generalizes the Misner-Sharp-Hernandez mass - better known from fluid dynamics in spherical symmetry [18] - and is universally adopted in black hole thermodynamics, but other constructs exist in the literature (see the review [19]).

Indeed, the quasilocal energy concept also appears in the first law of thermodynamics. Much literature has been devoted to horizon thermodynamics and to the ther-

\footnotetext{
* vfaraoni@ubishops.ca

$\dagger$ jcote16@ubishops.ca

1 As an example, bosonic string theory (the simplest string theory) reduces to an $\omega=-1$ Brans-Dicke theory, and not to GR, in the low-energy limit [2].
}

modynamics of gravity and spacetime in GR and in alternative theories. Thus far, very few quasilocal mass prescriptions have been given in scalar-tensor gravity, and they have important restrictions [20, 21]. For example, a certain prescription applies only to $f(\mathcal{R})$ gravity, or only to spherical symmetry, and sometimes only to very special spherical spacetime geometries. These prescriptions do not agree with each other and have been obtained in the context of the thermodynamics of spacetime. However, the definitions of four other quantities appearing in the first law (temperature, entropy, work density, and heat supply vector) are not established beyond doubt, which introduces uncertainty in any definition of quasilocal mass obtained by assuming a certain form of the first law. Additionally, the concept of temperature requires the study of quantum field theory in curved space, which is notoriously non-trivial, and where it is difficult to conclude calculations unambiguously (or to conclude them at all).

While we remain agnostic on these approaches based on the thermodynamics of spacetime, we have proposed an extension of the Hawking-Hayward quasilocal mass to scalar-tensor gravity that bypasses these difficulties using arguments that are purely classical and independent of thermodynamics [22]. The generalization of the Hawking-Hayward mass to scalar-tensor gravity is not restricted to $f(\mathcal{R})$ gravity or to special geometries, nor does it require spherical symmetry or asymptotic flatness (although we use the last two assumptions in our check of the validity of the quasilocal prescription in the present work).

This quasilocal mass generalization can be derived using two distinct approaches which give the same result in vacuo, but differ slightly in the presence of matter [22]. The first approach consists of writing the scalar-tensor field equations as effective Einstein equations and using the geometric derivation of the Hawking-Hayward mass. It uses minimal assumptions and should be regarded as the most reliable prescription [22]. The second approach relies on the Einstein frame formulation of scalar-tensor gravity, in which an omnipresent scalar field couples nonminimally to matter, while the rest of the action looks like the Einsten-Hilbert one. Because of this feature, the theory is not strictly GR and this method produces an extra spurious factor $\phi^{2}$ multiplying the matter contribution to the quasilocal mass (where $\phi$ is the Brans-Dicke scalar) [22]. 
In the present article, we propose a partial check of our prescription for the quasilocal mass in vacuo [22]. A minimal requirement for this prescription to make sense is that, in an asymptotically flat stationary geometry, the quasilocal mass reduces to the coefficient of the monopole term in a multipole expansion of the gravitational field. Such a multipole formalism for scalar-tensor gravity has been introduced recently in Ref. [23] for cylindrically symmetric and asymptotically flat systems. We show that the quasilocal mass computed at spatial infinity does indeed reduce to the coefficient of the lowest multipole, lending some confidence in the prescription of Ref. [22].

Let us begin by reviewing basic scalar-tensor gravity. The (Jordan frame) scalar-tensor action is [6, 8]

$$
\begin{aligned}
S_{\mathrm{ST}}= & \int d^{4} x \sqrt{-g}\left\{\left[\frac{1}{16 \pi}\left(\phi \mathcal{R}-\frac{\omega(\phi)}{\phi} g^{a b} \nabla_{a} \phi \nabla_{b} \phi\right)\right.\right. \\
& \left.-V(\phi)]+\mathcal{L}^{(\mathrm{m})}\right\},
\end{aligned}
$$

where $\mathcal{R}$ is the Ricci curvature of the spacetime metric $g_{a b}$ with determinant $g, \phi$ is the Brans-Dicke-like scalar field (roughly speaking, the inverse of the gravitational coupling strength which is varying in these theories [8]), $V(\phi)$ is a scalar field potential, and $\mathcal{L}^{(\mathrm{m})}$ is the matter Lagrangian density. The conformal rescaling of the metric

$$
g_{a b} \rightarrow \tilde{g}_{a b}=\Omega^{2} g_{a b}, \quad \Omega=\sqrt{\phi}
$$

and the scalar field redefinition $\phi \rightarrow \tilde{\phi}$ given by

$$
d \tilde{\phi}=\sqrt{\frac{2 \omega(\phi)+3}{16 \pi}} \frac{d \phi}{\phi}
$$

transform the action to its Einstein frame form

$$
\begin{aligned}
S_{\mathrm{ST}}= & \int d^{4} x \sqrt{-g}\left[\frac{\tilde{\mathcal{R}}}{16 \pi}-\frac{1}{2} \tilde{g}^{a b} \nabla_{a} \tilde{\phi} \nabla_{b} \tilde{\phi}-U(\tilde{\phi})\right. \\
& \left.+\frac{\mathcal{L}^{(\mathrm{m})}}{\phi^{2}}\right] .
\end{aligned}
$$

This is formally the action of GR with a scalar field coupling minimally to the curvature (but nonminimally to matter), where the scalar field has canonical kinetic energy density and

$$
U(\tilde{\phi})=\left.\frac{V(\phi)}{\phi^{2}}\right|_{\phi=\phi(\tilde{\phi})} .
$$

(A tilde identifies Einstein frame quantities.) Formally, the difference with GR is in the nonminimal (but universal) coupling of the Einstein frame scalar $\tilde{\phi}$ to (nonconformal) matter. This coupling becomes irrelevant in vacuo; otherwise, one can regard the scalar field $\tilde{\phi}$ and the matter fields described by $\mathcal{L}^{(\mathrm{m})}$ as mutually interacting forms of matter, with the condition that the scalar field $\tilde{\phi}$ is always present and cannot be switched off.
$f(\mathcal{R})$ theories of gravity, widely used to explain the present acceleration of the universe without dark energy [4, 5], are a subclass of scalar-tensor theories with action

$$
S_{f(\mathcal{R})}=\int d^{4} x \sqrt{-g} f(\mathcal{R})+S_{(\mathrm{m})}
$$

where $f(\mathcal{R})$ is a nonlinear function of the Ricci scalar. By setting $\phi=f^{\prime}(\mathcal{R})$ and

$$
V(\phi)=\phi \mathcal{R}(\phi)-f(\mathcal{R}(\phi)),
$$

the action can be shown to be equivalent to the scalartensor one [5]

$$
S=\int d^{4} x \frac{\sqrt{-g}}{16 \pi}[\phi \mathcal{R}-V(\phi)]+S_{(\mathrm{m})},
$$

which describes a Brans-Dicke theory with vanishing Brans-Dicke parameter $\omega$ and potential $V$ for the BransDicke scalar $\phi$.

\section{SPHERICAL SYMMETRY}

For simplicity, in our test of the scalar-tensor quasilocal prescription, we restrict to vacuum and spherical symmetry. With this symmetry, the Hawking-Hayward mass in GR reduces [24] to the better known Misner-SharpHernandez mass $\tilde{M}_{\mathrm{MSH}}$ defined by [18]

$$
1-\frac{2 \tilde{M}_{\mathrm{MSH}}}{\tilde{R}}=\tilde{g}^{a b} \nabla_{a} \tilde{R} \nabla_{b} \tilde{R}
$$

where $\tilde{R}$ is the areal radius of the spherical GR spacetime.

One of the two methods used in Ref. 22] to construct the scalar-tensor generalization of the Hawking-Hayward mass in the Jordan frame is by conformally transforming this mass to the Einstein frame and imposing that, in the Einstein frame, the usual GR expression holds and that this mass transforms as the Hawking-Hayward mass under a conformal mapping to the Jordan frame. Note that the second, and more reliable method, gives the same result in vacuo.

Under the conformal rescaling $g_{a b} \rightarrow \tilde{g}_{a b}=\Omega^{2} g_{a b}$, the Misner-Sharp-Hernandez mass transforms according to 25, 26,

$$
\tilde{M}_{\mathrm{MSH}}=\Omega M_{\mathrm{MSH}}-\frac{R^{3}}{2 \Omega} g^{a b} \nabla_{a} \Omega \nabla_{b} \Omega-R^{2} g^{a b} \nabla_{a} \Omega \nabla_{b} R .
$$

Specializing to the particular conformal transformation (1.2) and (1.3), Eq. (2.2) then yields the Jordan frame mass

$$
M_{\mathrm{JF}}=\frac{\tilde{M}_{\mathrm{MSH}}}{\sqrt{\phi}}+\frac{R^{3}}{8 \phi^{2}} g^{a b} \nabla_{a} \phi \nabla_{b} \phi+\frac{R^{2}}{2 \phi} g^{a b} \nabla_{a} \phi \nabla_{b} R,
$$

where all the quantities appearing on the right hand side, except $\tilde{M}_{\mathrm{MSH}}$, are Jordan frame quantities. In view of the 
consistency check presented below, however, it is convenient to re-express this formula in terms of Einstein frame quantities. Using the relations $\tilde{R}=\sqrt{\phi} R, g^{a b}=\phi \tilde{g}^{a b}$, and $\nabla_{a} \phi=\sqrt{\frac{16 \pi}{2 \omega+3}} \phi \nabla_{a} \tilde{\phi}$, one obtains

$$
\begin{aligned}
M_{\mathrm{JF}}= & \frac{\tilde{M}_{\mathrm{MSH}}}{\sqrt{\phi}}+\frac{2 \pi \phi R^{3}}{2 \omega+3} \tilde{g}^{a b} \nabla_{a} \tilde{\phi} \nabla_{b} \tilde{\phi} \\
& +\sqrt{\frac{16 \pi}{2 \omega+3}} \frac{R^{2}}{2} g^{a b} \nabla_{a} \tilde{\phi} \nabla_{b} R .
\end{aligned}
$$

Further, one notes that in spherical symmetry we have $\tilde{\phi}=\tilde{\phi}(\tilde{R})$, so $\nabla_{a} \tilde{\phi}=\frac{d \tilde{\phi}}{d \tilde{R}} \nabla_{a} \tilde{R}$. Then, one can use the chain rule and the definition $\tilde{R}=\sqrt{\phi} R$ to find

$$
\nabla_{a} R=\frac{\nabla_{a} \tilde{R}}{\sqrt{\phi}}\left(1-\frac{\tilde{R}}{2} \sqrt{\frac{16 \pi}{2 \omega+3}} \frac{d \tilde{\phi}}{d \tilde{R}}\right)
$$

Therefore, the last term in the definition of the mass transforms as

$$
\tilde{g}^{a b} \nabla_{a} \tilde{R} \nabla_{b} R=\frac{\nabla_{a} \tilde{R} \nabla_{b} \tilde{R}}{\sqrt{\phi}}\left(1-\frac{\tilde{R}}{2} \sqrt{\frac{16 \pi}{2 \omega+3}} \frac{d \tilde{\phi}}{d \tilde{R}}\right) .
$$

Putting these pieces together finally gives

$$
\begin{aligned}
M_{\mathrm{JF}}= & \frac{\tilde{M}_{\mathrm{MSH}}}{\sqrt{\phi}}+\frac{2 \tilde{R}^{2}}{\sqrt{\phi}} \sqrt{\frac{\pi}{2 \omega+3}}\left(\frac{d \tilde{\phi}}{d \tilde{R}}\right) \tilde{g}^{a b} \nabla_{a} \tilde{R} \nabla_{b} \tilde{R} \\
& \cdot\left[1-\sqrt{\frac{\pi}{2 \omega+3}}\left(\frac{d \tilde{\phi}}{d \tilde{R}}\right) \tilde{R}\right]
\end{aligned}
$$

\section{QUASILOCAL MASS AND MULTIPOLE EXPANSION}

A minimal requirement is that, for cylindrically symmetric and stationary geometries, when the mass $M_{\mathrm{JF}}$ is evaluated at spatial infinity $R \rightarrow+\infty$, it reduces to the monopole coefficient in a multipole expansion of the gravitational field. A multipole expansion for axisymmetric and asymptotically flat spacetimes in scalar-tensor gravity has been given recently by Pappas and Sotiriou 23. Their goal was to express observable quantities (such as the frequency of quasi-periodic oscillations and the frequency emitted near the inner boundary of accretion discs orbiting black holes in low-mass X-ray binaries) in terms of the multipole moments of spacetime, which is useful in tests of gravity. The general multipole formalism for scalar-tensor gravity is rather involved, but we can perform a simple check of Eq. (2.7) on a well-known and very general class of spacetimes (used also in [23] as a consistency check). This is the Just static spherically symmetric, asymptotically flat solution of scalar-tensor gravity [7, 27]. This 2-parameter class of solutions is the most general static, spherically symmetric, asymptotically flat solution of the vacuum Brans-Dicke field equations with vanishing mass or potential $(V(\phi)=0)$ which is not a black hole2 [28, 29]. When written in its Einstein frame representation, this solution is nothing but the Fisher solution of GR with a minimally coupled, free scalar field 3 [31, 32]. It is the most general static, spherical, and asymptotically flat solution of the Einstein equations with a free scalar field [32]. The Einstein frame line element and scalar field are

$$
\begin{aligned}
& d \tilde{s}^{2}=-V^{\nu}(r) d t^{2}+V^{-\nu}(r) d r^{2}+V^{1-\nu}(r) r^{2} d \Omega_{(2)}^{2} \\
& \tilde{\phi}(r)=\phi_{0} \ln V(r)+\phi_{1} \\
& V(r)=1-\frac{2 \mu}{r}
\end{aligned}
$$

where $d \Omega_{(2)}^{2}=d \theta^{2}+\sin ^{2} \theta d \varphi^{2}$ is the line element on the unit 2-sphere, $\phi_{0}, \phi_{1}, \mu$, and $\nu$ are constants and, because of asymptotic flatness, we set $\phi_{1}=1$. In the notation of Ref. 23] the exponent satisfies $\nu=m / l$, where $m$ and $l$ are length scales, and $\mu=l$. The areal radius of the Just geometry is clearly

$$
\tilde{R}=r\left(1-\frac{2 \mu}{r}\right)^{\frac{1-\nu}{2}}
$$

By applying the GR definition (2.1) in the Einstein frame, one obtains the Misner-Sharp-Hernandez mass of the Fisher spacetime

$$
\tilde{M}_{\mathrm{MSH}}=\frac{\tilde{R}}{2}\left\{1-\frac{1}{V(r)}\left[1-\frac{(\nu+1) \mu}{r}\right]^{2}\right\} .
$$

Since the multipoles have to be evaluated at spatial infinity, which corresponds to the limits $r, R, \tilde{R} \rightarrow+\infty$, one finds

$$
\tilde{M}_{\mathrm{MSH}} \simeq \nu \mu
$$

Then, using the relations [23] $\nu=m / l$ and $\mu=l$, one obtains $\tilde{M}_{\mathrm{MSH}}=m$.

We can now compute the scalar-tensor mass in the Jordan frame for this very general geometry using Eq. (2.7), and then take the limit to spatial infinity. First, we compute the following relation

\footnotetext{
2 The static spherical asymptotically flat black hole can only be the Schwarzschild black hole [30], for which the Misner-SharpHernandez mass trivially coincides with the Schwarzschild mass everywhere outside the horizon.

${ }^{3}$ This geometry has been rediscovered many times and is known by various names (Fisher-Janis-Newman-Winicour-BuchdahlWyman solution).
} 


$$
\begin{aligned}
\frac{d \tilde{\phi}}{d \tilde{R}} & =\frac{d \tilde{\phi}}{d r}\left(\frac{d \tilde{R}}{d r}\right)^{-1} \\
& =\frac{d}{d r}\left[\phi_{0} \ln V+\phi_{1}\right]\left\{V^{-\frac{(\nu+1)}{2}}\left[1-\frac{(\nu+1) \mu}{r}\right]\right\}^{-1} \\
& =\frac{2 \phi_{0} \mu V^{\frac{\nu-1}{2}}}{r^{2}\left[1-\frac{(\nu+1) \mu}{r}\right]}
\end{aligned}
$$

Then, one obtains the following for the quasilocal mass

$$
\begin{aligned}
M_{\mathrm{JF}}= & \frac{m}{\sqrt{\phi}}+\frac{4 \tilde{R}^{2}}{\sqrt{\phi}} \sqrt{\frac{\pi}{2 \omega+3}} \frac{\phi_{0} \mu V^{\frac{\nu-3}{2}}}{r^{2}} \\
& \cdot\left[1-\frac{\mu(1+\nu)}{r}-\sqrt{\frac{\pi}{2 \omega+3}} \frac{2 \phi_{0} \mu V^{\frac{\nu-1}{2}} \tilde{R}}{r^{2}}\right] .
\end{aligned}
$$

In the limit $r \rightarrow+\infty$, we have $\phi \rightarrow 1, V(r) \rightarrow 1$, and the quasilocal mass seen from spatial infinity is

$$
M_{\mathrm{JF}}^{(\mathrm{Just})} \simeq m+\sqrt{\frac{16 \pi}{2 \omega+3}} \phi_{0} l .
$$

Let us now compare our prescription w.9 with the monopole coefficient. The scalar-tensor multipole expansion of Ref. [23] gives the Jordan frame monopole coefficient

$$
M_{\mathrm{JF}}=m-w_{A} \alpha_{0}
$$

and the Einstein frame scalar field is written as

$$
\tilde{\phi}=\frac{w_{A}}{2 l} \ln \left(1-\frac{2 l}{r}\right),
$$

so $w_{A}=2 l \phi_{0}$. Ref. [23] uses the widespread notation [7] $A(\phi)=\Omega^{-1}=\phi^{-1 / 2}$, and $\alpha_{0} \equiv d(\ln A) /\left.d \tilde{\phi}\right|_{\infty}$. It is then straightforward to derive

$$
\alpha_{0}=-\left.\frac{1}{2 \phi} \frac{d \phi}{d \tilde{\phi}}\right|_{\infty}=-\sqrt{\frac{4 \pi}{2 \omega+3}}
$$

and

$$
M_{\mathrm{JF}}=m+4 l \phi_{0} \sqrt{\frac{\pi}{2 \omega+3}},
$$

which coincides with the expression (3.9) of $M_{\mathrm{JF}}^{(\mathrm{Just})}$. The quasilocal mass introduced in Ref. [22] reproduces (in the limit to spatial infinity) the monopole coefficient obtained in a multipole expansion which was performed entirely in the Jordan frame and is completely independent of our considerations.

\section{CONCLUSIONS}

As a partial check of the reliability of the quasilocal mass prescription of [22] in scalar-tensor gravity, we have shown that, in a very general vacuum situation, this construct reproduces (in the limit to spatial infinity) the monopole coefficient provided by the recent multipole expansion of Ref. [23]. This multipole formalism was derived entirely in the Jordan frame, in a way completely independent of our considerations. This fact inspires confidence in the recipe of Ref. 22] for extending the quasilocal mass to scalar-tensor gravity.

On the one hand, the extent of our check is limited because, in order to have a multipole expansion, one needs to restrict to stationary and asymptotically flat geometries and it is necessary to look at the physical system from spatial infinity. Moreover, a multipole expansion of the relativistic gravitational field is complicated and its implementation in scalar-tensor gravity is quite recent 23]. On the other hand, we have applied the quasilocal prescription to the most general static, spherically symmetric, and asymptotically flat solution of BransDicke theory with free scalar field that is not a black hole [28, 29]. All stable static, spherical, and asymptotically flat black hole solutions of (even more general) scalar-tensor theories reduce to the Schwarzschild black hole [30], and the quasilocal mass is then trivial.

Further tests of the quasilocal mass prescription of Ref. [22] and its application to horizon thermodynamics will be given in future work.

\section{ACKNOWLEDGMENTS}

This work is supported by the Natural Sciences and Engineering Research Council of Canada (Grant No. 2016-03803 to V.F.) and by Bishop's University.
[1] R. Utiyama and B.S. DeWitt, J. Math. Phys. 3, 608 (1962); K.S. Stelle, Gen. Relativ. Gravit. 9, 353 (1978); A. Giusti, Int. J. Geom. Meth. Mod. Phys. 16, 1930001 (2019); I.L. Buchbinder, S.D. Odintsov, and I.L. Shapiro, Effective Actions in Quantum Gravity (IOP, Bristol, 1992); G.A. Vilkovisky, Class. Quantum Grav. 9, 895 (1992).
[2] C.G. Callan, D. Friedan, E.J. Martinez, and M.J. Perry, Nucl. Phys. B 262, 593 (1985); E.S. Fradkin and A.A. Tseytlin, Nucl. Phys. B 261, 1 (1985).

[3] L. Amendola and S. Tsujikawa, Dark Energy, Theory and Observations (Cambridge University Press, Cambridge, 2010).

[4] S. Capozziello, S. Carloni, and A. Troisi, Re- 
cent Res. Dev. Astron. Astrophys. 1, 625 (2003) arXiv:astro-ph/0303041; S.M. Carroll, V. Duvvuri, M. Trodden, and M.S. Turner, Phys. Rev. D 70, 043528 (2004).

[5] T.P. Sotiriou and V. Faraoni, Rev. Mod. Phys. 82, 451 (2010); A. De Felice and S. Tsujikawa, Living Rev. Relativity 13, 3 (2010); S. Nojiri and S.D. Odintsov, Phys. Rept. 505, 59 (2011).

[6] P.G. Bergmann, Int. J. Theor. Phys. 1, 25 (1968); R.V. Wagoner, Phys. Rev. D 1, 3209 (1970); K. Nordtvedt, Astrophys. J. 161, 1059 (1970).

[7] T. Damour and G. Esposito-Farése, Phys. Rev. D 54, 1474 (1996).

[8] C.H. Brans and R.H. Dicke, Phys. Rev. 124, 925 (1961).

[9] C.M. Will, Theory and Experiment in Gravitational Physics (Cambridge University Press, Cambridge, 1993); C.M. Will, Living Rev. Relativ. 9, 3 (2006).

[10] E. Berti et al., Classical Quantum Gravity 32, 243001 (2015); T. Baker, D. Psaltis, and C. Skordis, Astrophys. J. 802, 63 (2015).

[11] T. Clifton, P.G. Ferreira, A. Padilla, and C. Skordis, Phys. Rep. 513, 1 (2012).

[12] T. Baker, D. Psaltis, and C. Skordis, Astrophys. J. 802, 63 (2015).

[13] K. Koyama, Rept. Prog. Phys. 79, 046902 (2016).

[14] L. Amendola et al., Living Rev. Relativity 16, 6 (2013); M. Ishak, Living Rev. Relativity 22, 1 (2019).

[15] K. Yagi and L.C. Stein, Classical Quantum Gravity 33, 054001 (2016); J. Sakstein, B. Jain, J.S. Heyl, and L. Hui, Astrophys. J. 844, L14 (2017); A.E. Broderick, T. Johannsen, A. Loeb, and D. Psaltis, Astrophys. J. 784, 7 (2014); R. Abuter et al. (GRAVITY collaboration), Astron. Astrophys. 615, L15 (2018).

[16] J.R. Gair, M. Vallisneri, S.L. Larson, and J.G. Baker, Living Rev. Relativity 16, 7 (2013); J. Sakstein and B. Jain, Phys. Rev. Lett. 119, 251303 (2017); P. Creminelli and F. Vernizzi, Phys. Rev. Lett. 119, 251302 (2017); T. Baker, E. Bellini, P.G. Ferreira, M. Lagos, J. Noller, and I. Sawicki, Phys. Rev. Lett. 119, 251301 (2017); J.M. Ezquiaga and M. Zumalacárregui, Phys. Rev. Lett. 119, 251301 (2017); L. Lombriser and A. Taylor, J. Cosmol.
Astropart. Phys. 1603, 31 (2016); L. Lombriser and N.A. Lima, Phys. Lett. B 765, 382 (2017).

[17] S. Hawking, J. Math. Phys. (N.Y.) 9, 598 (1968); S.A. Hayward, Phys. Rev. D 49, 831 (1994).

[18] C.W. Misner and D.H. Sharp, Phys. Rev. 136, B571 (1964); W.C. Hernandez and C.W. Misner, Astrophys. J. 143, 452 (1966).

[19] L.B. Szabados, Living Rev. Relativity 12, 4 (2009).

[20] R.-G. Cai, L.-M. Cao, Y.-P. Hu, and N. Ohta, Phys. Rev. D 80, 104016 (2009); R.-G. Cai, L.-M. Cao, Y.-P. Hu, and S.P. Kim, Phys. Rev. D 78, 124012 (2008); H. Zhang, Y. Hu, and X.-Z. Li, Phys. Rev. D 90, 024062 (2014).

[21] G. Cognola, O. Gorbunova, L. Sebastiani, and S. Zerbini, Phys. Rev. D 84, 023515 (2011).

[22] V. Faraoni, Class, Quantum Grav. 33, 015007 (2016).

[23] G. Pappas and T.P. Sotiriou, Phys. Rev. D 91, 044011 (2015); G. Pappas and T.P. Sotiriou, Mon. Not. Roy. Astron. Soc. 453, 2862 (2015).

[24] S.A. Hayward, Phys. Rev. D 53, 1938 (1996).

[25] A. Prain, V. Vitagliano, V. Faraoni, and M. LapierreLéonard, Class. Quantum Grav. 33, 145008 (2016).

[26] V. Faraoni and V. Vitagliano, Phys. Rev. D 89, 064015 (2014).

[27] K. Just, Zeitschrift Naturforschung Teil A 14, 751 (1959).

[28] K.A. Bronnikov, Acta Phys. Pol. B 4, 251 (1973).

[29] V. Faraoni, F. Hammad, A.M. Cardini, and T. Gobeil, Phys. Rev. D 97, 084033 (2018).

[30] S.W. Hawking, Comm. Math. Phys. 25, 167 (1972); J.D. Bekenstein, arXiv:gr-qc/9605059, T.P. Sotiriou and V. Faraoni, Phys. Rev. Lett. 108, 081103 (2012); S. Bhattacharya, K.F. Dialektopoulos, A.E. Romano, and T.N. Tomaras, Phys. Rev. Lett. 115, 181104 (2015); V. Faraoni, Phys. Rev. D 95, 124013 (2017).

[31] I.Z. Fisher, Zh. Eksp. Teor. Fiz. 18, 636 (1948) arXiv:gr-qc/9911008; O. Bergman and R. Leipnik, Phys. Rev. 107, 1157 (1957); A.I. Janis, E.T. Newman, and J. Winicour, Phys. Rev. Lett. 20, 878 (1968); H.A. Buchdahl, Int. J. Theor. Phys. 6, 407 (1972).

[32] M. Wyman, Phys. Rev. D 24, 839 (1981). 